\title{
Two-sided non-collapsing curvature flows
}

\author{
BEN ANDREWS AND MAT LANGFORD
}

\begin{abstract}
It was recently shown that embedded solutions of curvature flows in Euclidean space with concave (convex), degree one homogeneous speeds are interior (exterior) non-collapsing [6]. These results were subsequently extended to hypersurface flows in the sphere and hyperbolic space [11]. In the first part of the paper, we show that locally convex solutions are exterior non-collapsing for a larger class of speed functions than previously considered; more precisely, we show that the previous results hold when convexity of the speed function is relaxed to inverse-concavity. We note that inverse-concavity is satisfied by a large class of concave speed functions [4]. Thus, as a consequence, we obtain a large class of two-sided non-collapsing flows, whereas previously two-sided non-collapsing was only known for the mean curvature flow. In Section 3, we demonstrate the utility of two sided non-collapsing with a straightforward proof of convergence of compact, convex hypersurfaces to round points. The proof of the non-collapsing estimate is similar to those of the previous results mentioned, in that we show that the exterior ball curvature is a viscosity supersolution of the linearised flow equation. The new ingredient is the following observation: Since the function which provides an upper support in the derivation of the viscosity inequality is defined on $M \times M$ (or $T M$ in the 'boundary case'), whereas the exterior ball curvature and the linearised flow equation depend only on the first factor, we are privileged with a freedom of choice in which second derivatives from the extra directions to include in the calculation. The optimal choice is closely related to the class of inverse-concave speed functions.
\end{abstract}

Mathematics Subject Classification (2010): 53C44 (primary); 35K55, 58J35 (secondary).

\section{Introduction}

We consider embedded solutions $X: M^{n} \times[0, T) \rightarrow N_{\sigma}^{n+1}$ of curvature flows of the form

$$
\partial_{t} X(x, t)=-F(x, t) v(x, t)
$$

Research partially supported by Discovery grant DP120100097 of the Australian Research Council. The second author gratefully acknowledges the support of an Australian Postgraduate Award and an Australian National University HDR Supplementary Scholarship, and the support and hospitality of the Mathematical Sciences Center at Tsinghua University, Beijing, and the Department of Mathematics at East China Normal University, Shanghai during the completion of parts of this work.

Received October 2, 2013; accepted in revised form June 18, 2014.

Published online February 2016. 
where $N_{\sigma}^{n+1}$ is the complete, simply connected Riemannian manifold of constant curvature $\sigma \in\{-1,0,1\}$ (that is, either hyperbolic space $H^{n+1}$, Euclidean space $\mathbb{R}^{n+1}$, or the sphere $S^{n+1}$ ), $v$ is a choice of normal field for the evolving hypersurface $X$, and the speed $F$ is given by a smooth, symmetric, degree one homogeneous function of the principal curvatures $\kappa_{i}$ of $X$ which is monotone increasing with respect to each $\kappa_{i}$. Equivalently, $F$ is a smooth, monotone increasing, degree one homogeneous function of the Weingarten map $\mathcal{W}$ of $X$. Moreover, we will always assume that $F$ is normalised such that $F(1, \ldots, 1)=1$; however, this is merely a matter of convenience - all of the results hold, up to a recalibration of constants, in the un-normalised case.

The interior and exterior ball curvatures of a family of embeddings $X: M^{n} \times$ $[0, T) \rightarrow \mathbb{R}^{n+1}$ with normal $v$ are, respectively, defined by $\bar{k}(x, t):=\sup _{y \neq x} k(x, y, t)$ and $\underline{k}(x, t):=\inf _{y \neq x} k(x, y, t)$, where

$$
k(x, y, t):=\frac{2\langle X(x, t)-X(y, t), v(x, t)\rangle}{\|X(x, t)-X(y, t)\|^{2}} .
$$

Equivalently, $\bar{k}(x, t)$ (respectively $\underline{k}(x, t))$ gives the curvature of the largest region in $\mathbb{R}^{n+1}$ with totally umbilic boundary that lies on the opposite (respectively same) side of the hypersurface $X(M, t)$ into which $v(x, t)$ points, and touches the hypersurface at $X(x, t)$ (with sign determined by $v)$ [6, Proposition 4]. Therefore, for a compact, convex embedding with outward normal, they are, respectively, the curvature of the largest enclosed and smallest enclosing spheres which touch the embedding at $X(x, t)$. It follows that $\kappa_{\max } \leq \bar{k}$ and $\kappa_{\min } \geq \underline{k}$.

The interior and exterior ball curvatures (introduced by the authors and McCoy in [6]) are motivated by work of Sheng and Wang [23] who, via a detailed analysis of singularities, were able to prove that compact embedded solutions of the mean curvature flow in Euclidean space are interior non-collapsing: There exists a constant $K_{0}<\infty$ such that the inequality $\bar{k} \leq K_{0} H$ holds at all points and times. Motivated by this result, the first author found an elementary maximum principle proof of the interior non-collapsing estimate, which, in addition, yields an analogous exterior non-collapsing estimate [5]. Interior and exterior non-collapsing estimates were proved for large classes of Euclidean flows by the authors and McCoy [6]: We found that embedded, positive $(F>0)$ solutions of $(\mathrm{CF})$ are interior non-collapsing when the speed is a concave function of the Weingarten map and exterior non-collapsing when the speed is a convex function of the Weingarten map [6]. It was subsequently shown that non-collapsing estimates also hold when the ambient space is either the sphere or hyperbolic space [11]: Considering the sphere $S^{n+1}$ as the embedded submanifold $\left\{X \in \mathbb{R}^{n+2}:\langle X, X\rangle=1\right\}$ of $\mathbb{R}^{n+2}$, and hyperbolic space $H^{n+1}$ as the embedded submanifold $\left\{X \in \mathbb{R}^{n+1,1}:\langle X, X\rangle=-1\right\}$ of Minkowski space $\mathbb{R}^{n+1,1}$, the function $k$ may be formally defined by (1.1), except that now we take $\langle\cdot, \cdot\rangle$ and $\|\cdot\|$ to be the inner product and induced norm on, in the case of the sphere, $\mathbb{R}^{n+2}$, and, in the case of hyperbolic space, the spacelike vectors in $\mathbb{R}^{n+1,1}$. Then, if $F$ is a concave function of the curvatures, there exists 
$K_{0}>0$ such that

$$
\frac{\bar{k}}{F}-\frac{1}{\eta} \leq K_{0} \mathrm{e}^{-2 \sigma \eta t},
$$

and, if $F$ is a convex function of the curvatures, there exists $k_{0} \in \mathbb{R}$ such that

$$
\frac{\underline{k}}{F}-\frac{1}{\eta} \geq k_{0} \mathrm{e}^{-2 \sigma \eta t},
$$

where $\eta>0$ depends on bounds for the derivative of $F$. In this article, we extend the exterior non-collapsing estimate to flows by a larger class of speeds; namely, those by inverse-concave speeds:

Definition 1.1. Let $\Gamma_{+}$denote the positive cone in $\mathbb{R}^{n}: \Gamma_{+}:=\left\{z \in \mathbb{R}^{n}: z_{i}>\right.$ 0 for all $i\}$. A function $f: \Gamma_{+} \rightarrow \mathbb{R}$ is called inverse-concave if the function $f_{*}: \Gamma_{+} \rightarrow \mathbb{R}$ defined by $f_{*}\left(z_{1}^{-1}, \ldots, z_{n}^{-1}\right):=f\left(z_{1}, \ldots, z_{n}\right)^{-1}$ is concave.

Our main result may now be stated as follows:

Theorem 1.2. Let $f: \Gamma_{+} \rightarrow \mathbb{R}$ be a smooth, symmetric function which is homogeneous of degree one and monotone increasing in each argument. Let $X$ be a solution of (CF) with speed given by $F=f\left(\kappa_{1}, \ldots, \kappa_{n}\right)$, where $\kappa_{i}$ are the principal curvatures of $X$. Then, if $f$ is inverse-concave, $X$ is exterior non-collapsing; that is:

(1) If $N^{n+1}=\mathbb{R}^{n+1}$, then, for all $(x, t) \in M \times[0, T)$,

$$
\frac{\underline{k}(x, t)}{F(x, t)} \geq \inf _{M \times\{0\}} \frac{\underline{k}}{F}
$$

(2) If $N^{n+1}=S^{n+1}$ and $\operatorname{tr}(\dot{F}) \leq \eta$, then, for all $(x, t) \in M \times[0, T)$,

$$
\frac{\underline{k}(x, t)}{F(x, t)}-\frac{1}{\eta} \geq \inf _{M \times\{0\}}\left(\frac{\underline{k}}{F}-\frac{1}{\eta}\right) \mathrm{e}^{-2 \eta t} ;
$$

(3) If $N^{n+1}=H^{n+1}$ and $\operatorname{tr}(\dot{F}) \geq \eta$, then, for all $(x, t) \in M \times[0, T)$,

$$
\frac{\underline{k}(x, t)}{F(x, t)}-\frac{1}{\eta} \geq \inf _{M \times\{0\}}\left(\frac{k}{F}-\frac{1}{\eta}\right) \mathrm{e}^{2 \eta t},
$$

where $\dot{F}$ is the derivative of $F$ with respect to the Weingarten map.

In particular, combining Theorem 1.2 with the previous non-collapsing results [6, 11], we find that solutions of flows in spaceforms by concave, inverse-concave speed functions are both interior and exterior non-collapsing. We note that concave speed functions satisfy $\operatorname{tr}(\dot{F}) \geq 1$, so in that case we may take $\eta=1$ in case (3) of Theorem 1.2. 
We note that, for speeds defined on the positive cone, inverse-concavity is implied by convexity. Moreover, the class of admissible speeds which are both concave and inverse-concave is surprisingly large, including, for example, the degree one homogeneous roots of ratios of the elementary symmetric polynomials [4].

Two-sided non-collapsing has many useful consequences; for example, for uniformly convex hypersurfaces we obtain uniform pointwise bounds on the ratios of principal curvatures and a uniform bound on the ratio of circumradius to in-radius. We will show, in Section 3, how this leads to a new and simple proof that compact, convex hypersurfaces shrink to round points under flows by concave, inverse-concave speeds [4] (in particular, this applies to the mean curvature flow, yielding a new and simple proof of Huisken's theorem [20]). Moreover, onesided non-collapsing can also provide useful information; for example, interior noncollapsing rules out certain 'collapsing' singularity models, such as products of the Grim Reaper solution of the curve shortening flow with $\mathbb{R}^{n+1}$. For flows by convex speeds, exterior non-collapsing is sufficient to obtain a bound on the ratio of circumradius to in-radius, and the proof of convergence of locally convex initial hypersurfaces to round points [2] is also simplified.

ACKNOWLEDGEMENTS. The authors wish to express their thanks to Chen Xuzhong and Yong Wei for their helpful comments and suggestions on earlier versions of this work.

\section{Proof of Theorem 1.2}

We first extend $(c f \cdot[6]) k(\cdot, \cdot, t)$ to a continuous function on a compact manifoldwith-boundary, $\widehat{M}$. As a set, $\widehat{M}:=(M \times M \backslash D) \sqcup S M$, where $D:=\{(x, x)$ : $x \in M\}$ is the diagonal submanifold and $S M$ is the unit tangent bundle with respect to the metric at time $t$. The manifold-with-boundary structure is defined by the atlas generated by all charts for $(M \times M) \backslash D$, together with the charts $\widehat{Y}$ defined by $\widehat{Y}(z, s):=(\exp (s Y(z)), \exp (-s Y(z)))$ for $s$ sufficiently small, where $Y$ is a chart for $S M$. The extension is then given by setting $k(x, y, t):=\mathcal{W}_{(x, t)}(y, y)$ for $(x, y) \in S_{(x, t)} M$.

We also recall some useful notation from [5] and [6]; namely, we define

$$
d(x, y, t):=\|X(x, t)-X(y, t)\| \quad \text { and } \quad w(x, y, t):=\frac{X(x, t)-X(y, t)}{\|X(x, t)-X(y, t)\|},
$$

and use scripts $x$ and $y$ to denote quantities pulled back to $M \times M$ by the respective projections onto the first and second factor. With this notation in place, $k$ may be written as

$$
k=\frac{2}{d^{2}}\left\langle d w, v_{x}\right\rangle .
$$

Theorem 1.2 is a direct consequence of the following proposition: 
Proposition 2.1. If the flow speed $F$ is inverse-concave, then the exterior ball curvature $\underline{k}$ is a viscosity supersolution of the equation

$$
\partial_{t} u=\mathscr{L} u+\left(|\mathcal{W}|_{F}^{2}-\sigma \operatorname{tr}(\dot{F})\right) u+2 \sigma F,
$$

where $\mathscr{L}:=\dot{F}^{i j} \nabla_{i} \nabla_{j},\langle u, v\rangle_{F}:=\dot{F}^{i j} v_{i} v_{j}$, and $|\mathcal{W}|_{F}^{2}:=\dot{F}^{i j} \mathcal{W}_{i j}^{2}$.

We note that the speed function satisfies the equation

$$
\partial_{t} F=\mathscr{L} F+\left(|\mathcal{W}|_{F}^{2}+\sigma \operatorname{tr}(\dot{F})\right) F
$$

under the flow [3].

Proof of Proposition 2.1. Consider, for an arbitrary point $\left(x_{0}, t_{0}\right) \in M \times[0, T)$, an arbitrary lower support funtion $\phi$ for $\underline{k}$ at $\left(x_{0}, t_{0}\right)$; that is, $\phi$ is $C^{2,1}$ on a backwards parabolic neighbourhood $P:=U_{x_{0}} \times\left(t_{0}-\varepsilon, t_{0}\right]$ of $\left(x_{0}, t_{0}\right)$ and $\phi \leq \underline{k}$ with equality at $\left(x_{0}, t_{0}\right)$. Then we need to prove that the differential inequality

$$
\partial_{t} \phi \geq \mathscr{L} \phi+\left(|\mathcal{W}|_{F}^{2}-\sigma \operatorname{tr}(\dot{F})\right) \phi+2 \sigma F
$$

holds at $\left(x_{0}, t_{0}\right)$.

We note that $k(x, y, t) \geq \underline{k}(x, t) \geq \phi(x, t)$ for all $(x, y, t) \in \widehat{M} \times[0, T)$ such that $(x, t) \in P$, and, since $\bar{k}$ is continuous and $\widehat{M}$ is compact, we either have $\underline{k}\left(x_{0}, t_{0}\right)=k\left(x_{0}, y_{0}, t_{0}\right)$ for some $y_{0} \in M \backslash\left\{x_{0}\right\}$, or $\underline{k}\left(x_{0}, t_{0}\right)=\mathcal{W}_{\left(x_{0}, t_{0}\right)}\left(y_{0}, y_{0}\right)$ for some $y_{0} \in S_{\left(x_{0}, t_{0}\right)} M$. We consider the former case first.

\section{The interior case}

We first suppose that $\inf _{M} k\left(x_{0}, \cdot, t_{0}\right)<k\left(x_{0}, y_{0}, t_{0}\right)$ for all boundary points $\left(x_{0}, y_{0}\right)$ of $\widehat{M}$. In that case, we have $\kappa_{1}\left(x_{0}, t_{0}\right)>\underline{k}\left(x_{0}, t_{0}\right)=k\left(x_{0}, y_{0}, t_{0}\right)$ for some $y_{0} \in$ $M \backslash\left\{x_{0}\right\}$ and $k(x, y, t) \geq \underline{k}(x, t) \geq \phi(x, t)$ for all $(x, t) \in P$ and all $y \in M \backslash\{x\}$. In particular, we have the inequalities

$$
\text { and } \begin{aligned}
\partial_{t}(k-\phi) & \leq 0, \\
\widehat{\mathscr{L}}(k-\phi) & \geq 0
\end{aligned}
$$

at $\left(x_{0}, y_{0}, t_{0}\right)$ for any elliptic operator $\widehat{\mathscr{L}}$ on $M \times M$. We would like $\widehat{\mathscr{L}}$ to project to $\mathscr{L}$ on the first factor. This leads us to consider operators of the form $\widehat{\mathscr{L}}=$ $\dot{F}_{x}^{i j} \nabla_{\partial_{x_{i}}+\Lambda_{i}{ }^{p} \partial_{y} p} \nabla_{\partial_{x} j+\Lambda_{j} q} \partial_{y} q$, where $\Lambda$ is any $n \times n$ matrix.

We note that, in both of the cases $\sigma= \pm 1$, the ambient Euclidean/Minkowskian derivative decomposes into tangential and normal components as $D=\bar{D}-\bar{g} \otimes \bar{v}$, where $\bar{D}, \bar{g}$, and $\bar{v}$ are, respectively, the induced connection, metric, and outer/ future-pointing normal of $N_{\sigma}^{n+1}$ with respect to its embedding. Using the fact that 
$\langle\bar{\nu}, \bar{v}\rangle=\sigma$ and that the ambient position vector is normal to $N_{\sigma}^{n+1}$, a straightforward computation yields

$$
\left(\partial_{x^{i}}+\Lambda_{i}^{p} \partial_{y} p\right) k=\frac{2}{d^{2}}\left(\left\langle\partial_{i}^{x}-\Lambda_{i}^{p} \partial_{p}^{y}, v_{x}-k d w\right\rangle+\left\langle d w, \mathcal{W}_{i}^{x}{ }_{i}^{p} \partial_{p}^{x}\right\rangle\right) .
$$

If we choose the coordinates $\left\{x^{i}\right\}_{i=1}^{n}$ and $\left\{y^{i}\right\}_{i=1}^{n}$ to be orthonormal coordinates (with respect to the induced metric $g$ at time $t_{0}$ ) centred at $x_{0}$ and $y_{0}$ respectively, then a further straightforward computation using the vanishing of (2.3) at $\left(x_{0}, y_{0}, t_{0}\right)$ and the Codazzi equation yields

$$
\begin{aligned}
\nabla_{\partial^{j}}+\Lambda_{j}{ }^{q} \partial_{y} q & \nabla_{\partial_{x^{i}}+\Lambda_{i}{ }^{p} \partial_{y} p} k \\
= & \frac{2}{d^{2}}\left\{\left\langle-\mathcal{W}^{x}{ }_{i j} v_{x}-\sigma \delta_{i j} X_{x}+\Lambda_{i}{ }^{p} \Lambda_{j}{ }^{q}\left(\mathcal{W}^{y}{ }_{p q} v_{y}+\sigma \delta_{p q} X_{y}\right), v_{x}-k d w\right\rangle\right. \\
& +\left\langle\partial_{i}^{x}-\Lambda_{i}{ }^{p} \partial_{p}^{y}, \mathcal{W}^{x}{ }_{j}{ }^{q} \partial_{q}^{x}\right\rangle-\left(\partial_{x^{j}}+\Lambda_{j}{ }^{q} \partial_{y^{q}}\right) k\left\langle\partial_{i}^{x}-\Lambda_{i}{ }^{p} \partial_{p}^{y}, d w\right\rangle \\
& -k\left\langle\partial_{i}^{x}-\Lambda_{i}{ }^{p} \partial_{p}^{y}, \partial_{j}^{x}-\Lambda_{j}{ }^{q} \partial_{q}^{y}\right\rangle+\left\langle\partial_{j}^{x}-\Lambda_{j}{ }^{q} \partial_{q}^{y}, \mathcal{W}^{x}{ }_{i}{ }^{p} \partial_{p}^{x}\right\rangle \\
& +\left\langle d w, \nabla \mathcal{W}^{x}{ }_{i j}-\sigma \mathcal{W}^{x}{ }_{i j} X_{x}-\mathcal{W}^{x}{ }_{i}{ }^{r} \mathcal{W}^{x}{ }_{r j} v_{x}\right\rangle \\
& \left.-\left(\partial_{x^{i}}+\Lambda_{i}{ }^{p} \partial_{y} p\right) k\left\langle\partial_{j}^{x}-\Lambda_{j}{ }^{q} \partial_{q}^{y}, d w\right\rangle\right\}
\end{aligned}
$$

at the point $\left(x_{0}, y_{0}, t_{0}\right)$.

Next, noting that the normal satisfies $D_{t} v=\bar{D}_{t} v+\sigma F X=\operatorname{grad} F+\sigma F X$, we compute

$$
\partial_{t} k=\frac{2}{d^{2}}\left(\left\langle-F_{x} v_{x}+F_{y} v_{y}, v_{x}-k d w\right\rangle+\left\langle d w, \operatorname{grad} F_{x}+\sigma F X_{x}\right\rangle\right) .
$$

Combining (2.4) and (2.5), we obtain

$$
\begin{aligned}
\left(\partial_{t}-\widehat{\mathscr{L}}\right) k= & \frac{2}{d^{2}}\left\{\left\langle F_{y} v_{y}-\dot{F}_{x}^{i j} \Lambda_{i}{ }^{p} \Lambda_{j}^{q}\left(\mathcal{W}^{y}{ }_{p q} v_{y}+\sigma \delta_{p q} X_{y}\right), v_{x}-k d w\right\rangle\right. \\
& +k \dot{F}_{x}^{i j}\left\langle\partial_{i}^{x}-\Lambda_{i}{ }^{p} \partial_{p}^{y}, \partial_{j}^{x}-\Lambda_{j}^{q} \partial_{q}^{y}\right\rangle-2 \dot{F}_{x}^{i j}\left\langle\partial_{j}^{x}-\Lambda_{j}{ }^{q} \partial_{q}^{y}, \mathcal{W}^{x}{ }_{i}{ }^{p} \partial_{p}^{x}\right\rangle \\
& \left.+\sigma \operatorname{tr}\left(\dot{F}_{x}\right)\left\langle X_{x}, v_{x}-k d w\right\rangle+2 \sigma F_{x}\left\langle X_{x}, d w\right\rangle\right\} \\
& +\frac{4}{d^{2}} \dot{F}_{x}^{i j} \nabla_{\partial_{x^{i}}+\Lambda_{i}{ }^{p}{ }_{\partial_{y}} k} k\left\langle\partial_{j}^{x}-\Lambda_{j}{ }^{q} \partial_{q}^{y}, d w\right\rangle+\left|\mathcal{W}^{x}\right|_{F}^{2} k
\end{aligned}
$$

at the point $\left(x_{0}, y_{0}, t_{0}\right)$.

We now note that the vanishing of the $y$-derivatives at an off-diagonal extremum $y_{0} \in M$ of $k\left(x_{0}, \cdot, t_{0}\right)$ determines the tangent plane to $X$ at $y_{0}$ :

Lemma $2.2([6,11])$. Suppose that a point $(x, y, t)$ is an off-diagonal extremum of $k$; that is, $y \neq x$ is an extremum of $k(x, \cdot, t)$. Then

$$
\operatorname{span}\left\{\partial_{i}^{x}-2\left\langle\partial_{i}^{x}, w\right\rangle w\right\}_{i=1}^{n}=\operatorname{span}\left\{\partial_{i}^{y}\right\}_{i=1}^{n}
$$

at $(x, y, t)$, where $\left\{\partial_{i}^{x}\right\}_{i=1}^{n}$ and $\left\{\partial_{i}^{y}\right\}$ are bases for $T_{x} M$ and $T_{y} M$ respectively. 
Proof of Lemma 2.2. We may assume that $\left\{\partial_{i}^{x}\right\}_{i=1}^{n}$ and $\left\{\partial_{i}^{y}\right\}_{i=1}^{n}$ are orthonormal. Then $\left\{\partial_{i}^{x}-2\left\langle\partial_{i}^{x}, w\right\rangle w\right\}_{i=1}^{n}$ is also orthonormal; note also that $\left\|v_{x}-k d w\right\|=1$. Next, observe that the vanishing of $\partial_{y^{i}} k$ implies

$$
\left\langle\partial_{i}^{y}, v_{x}-k d w\right\rangle=0
$$

for each $i$. If $\sigma \neq 0$, a short computation, using $d^{2}=2\left(\sigma-\left\langle X_{x}, X_{y}\right\rangle\right)$, yields

$$
\left\langle X_{y}, v_{x}-k d w\right\rangle=0 .
$$

Thus, the orthogonal complement of $\operatorname{span}\left\{\partial_{i}^{y}\right\}_{i=1}^{n}$ is $\operatorname{span}\left\{\sigma X_{y}, v_{x}-k d w\right\}$. On the other hand, one easily computes

$$
\left\langle\partial_{i}^{x}-2\left\langle\partial_{i}^{x}, w\right\rangle w, v_{x}-k d w\right\rangle=0
$$

for each $i$, and, for $\sigma \neq 0$,

$$
\left\langle\partial_{i}^{x}-2\left\langle\partial_{i}^{x}, w\right\rangle w, X_{y}\right\rangle=0 .
$$

Thus, $\operatorname{span}\left\{\partial_{i}^{x}-2\left\langle\partial_{i}^{x}, w\right\rangle w\right\}^{\perp}=\operatorname{span}\left\{\sigma X_{y}, v_{x}-k d w\right\}$. The claim follows.

Thus, without loss of generality, we may assume

$$
\partial_{i}^{y}=\partial_{i}^{x}-2\left\langle\partial_{i}^{x}, w\right\rangle w
$$

at $\left(x_{0}, y_{0}, t_{0}\right)$. Note also that, when $\sigma \neq 0$,

$$
\left.\frac{2}{d^{2}}\left\langle X_{x}, v_{x}-k d w\right\rangle\right|_{\left(x_{0}, y_{0}, t_{0}\right)}=\left.\frac{2}{d^{2}}\left\langle X_{x}-X_{y}, v_{x}-k d w\right\rangle\right|_{\left(x_{0}, y_{0}, t_{0}\right)}=-k\left(x_{0}, y_{0}, t_{0}\right) .
$$

Finally, observe that (2.3) implies

$$
\frac{2}{d^{2}}\left\langle d w, \partial_{i}^{x}\right\rangle=R_{i}^{p} \partial_{x} p k
$$

where we have defined the (positive definite) map $R:=\left(\mathcal{W}^{x}-k I\right)^{-1}$, with $I$ : $T_{x} M \rightarrow T_{x} M$ denoting the identity map.

Using these observations and the vanishing of $\partial_{y^{i}} k$, we obtain

$$
\begin{aligned}
\left(\partial_{t}-\widehat{\mathscr{L}}\right) k= & \left(\left|\mathcal{W}^{x}\right|_{F}^{2}-\sigma \operatorname{tr}\left(\dot{F}_{x}\right)\right) k+2 \sigma F_{x}+2 \dot{F}_{x}^{i j} \partial_{x^{i}} k R_{j}{ }^{p} \partial_{x} p k \\
& +\frac{2}{d^{2}}\left\{F_{y}-F_{x}+\dot{F}_{x}^{i j}\left[\left(k \delta_{i j}-\mathcal{W}^{x}{ }_{i j}\right)\right.\right. \\
& \left.\left.-2 \Lambda_{i}{ }^{p}\left(k \delta_{p j}-\mathcal{W}^{x}{ }_{p j}\right)+\Lambda_{i}{ }^{p} \Lambda_{j}{ }^{q}\left(k \delta_{p q}-\mathcal{W}^{y}{ }_{p q}\right)\right]\right\}
\end{aligned}
$$

at any off-diagonal extremum $\left(x_{0}, y_{0}, t_{0}\right)$. 
Applying the inequalities (2.2), we obtain

$$
\begin{aligned}
0 \geq & \left(\partial_{t}-\widehat{\mathscr{L}}\right)(k-\phi) \\
\geq & -\left(\partial_{t}-\mathscr{L}\right) \phi+\left(\left|\mathcal{W}^{x}\right|_{F}^{2}-\sigma \operatorname{tr}\left(\dot{F}_{x}\right)\right) k+2 \sigma F_{x}+2 \dot{F}_{x}^{i j} \partial_{i} k R_{j}{ }^{p} \partial_{p} k \\
& +\frac{2}{d^{2}}\left\{F_{y}-F_{x}+\dot{F}_{x}^{i j}\left[\left(k \delta_{i j}-\mathcal{W}^{x}{ }_{i j}\right)-2 \Lambda_{i}{ }^{p}\left(k \delta_{p j}-\mathcal{W}_{p j}^{x}\right)\right.\right. \\
& \left.\left.+\Lambda_{i}{ }^{p} \Lambda_{j}{ }^{q}\left(k \delta_{p q}-\mathcal{W}^{y}{ }_{p q}\right)\right]\right\} .
\end{aligned}
$$

It remains to demonstrate non-negativity of the term on the second line for some choice of the matrix $\Lambda$. Since we are free to choose the orthonormal basis at $y_{0}$ such that $\mathcal{W}$ is diagonalised, this follows from the following proposition.

Proposition 2.3. Let $f: \Gamma_{+} \rightarrow \mathbb{R}$ be a smooth, symmetric function which is monotone increasing in each variable and inverse-concave, and let $F: \mathcal{C}_{+} \rightarrow \mathbb{R}$ be the function defined on the cone $\mathcal{C}_{+}$of positive definite symmetric matrices by $F(A)=f(\lambda(A))$, where $\lambda$ denotes the eigenvalue map. Then, for any $k \in \mathbb{R}$, any diagonal $B \in \mathcal{C}_{+}$, and any $A \in \mathcal{C}_{+}$with $k<\min _{i}\left\{\lambda_{i}(A)\right\}$, we have

$$
\begin{aligned}
0 \leq & F(B)-F(A)+\dot{F}^{i j}(A) \sup _{\Lambda}\left[\left(k \delta_{i j}-A_{i j}\right)-2 \Lambda_{i}{ }^{p}\left(k \delta_{p j}-A_{p j}\right)\right. \\
& \left.+\Lambda_{i}{ }^{p} \Lambda_{j}{ }^{q}\left(k \delta_{p q}-B_{p q}\right)\right] .
\end{aligned}
$$

Proof of Proposition 2.3. Since the expression in the square brackets is quadratic in $\Lambda$, it is easy to see that the supremum is attained with the choice $\Lambda=(A-k I)$. $(B-k I)^{-1}$, where $I$ denotes the identity matrix. Thus, given any $A \in \mathcal{C}_{+}$, we need to show that

$$
\begin{aligned}
0 \leq & Q_{A}(B):=F(B)-F(A) \\
& -\dot{F}^{i j}(A)\left((A-k I)_{i j}-\left[(A-k I) \cdot(B-k I)^{-1} \cdot(A-k I)\right]_{i j}\right) .
\end{aligned}
$$

Since $B$ is diagonal and the expression $Q_{A}(B)$ is invariant under similarity transformations with respect to $A$, we may diagonalise $A$ to obtain

$$
Q_{A}(B):=f(b)-f(a)-\dot{f}^{i}(a)\left[\left(a_{i}-k\right)-\frac{\left(a_{i}-k\right)^{2}}{b_{i}-k}\right],
$$

where we have set $a=\lambda(A)$ and $b=\lambda(B)$.

We are led to consider the function $q_{a}$ defined on $\Gamma_{+}$by

$$
q_{a}(z):=f(z)-f(a)-\dot{f}^{i}(a)\left[\left(a_{i}-k\right)-\frac{\left(a_{i}-k\right)^{2}}{z_{i}-k}\right] .
$$


We compute

$$
\dot{q}_{a}^{i}=\dot{f}^{i}-\dot{f}^{i}(a) \frac{\left(a_{i}-k\right)^{2}}{\left(z_{i}-k\right)^{2}},
$$

and

$$
\ddot{q}_{a}^{i j}=\dddot{f}^{i j}+2 \dot{f}^{i}(a) \frac{\left(a_{i}-k\right)^{2}}{\left(z_{i}-k\right)^{3}} \delta^{i j}=\dddot{f}^{i j}+2 \frac{\dot{f}^{i} \delta^{i j}}{z_{i}-k}-2 \frac{\dot{q}_{a}^{i} \delta^{i j}}{z_{i}-k} .
$$

It follows that

$$
\ddot{q}_{a}^{i j}+2 \frac{\dot{q}_{a}^{i} \delta^{i j}}{z_{i}-k}=\dddot{f}^{i j}+2 \frac{\dot{f}^{i} \delta^{i j}}{z_{i}-k}>\dddot{f}^{i j}+2 \frac{\dot{f}^{i} \delta^{i j}}{z_{i}} \geq 0,
$$

where the last inequality follows from inverse-concavity of $f$ [4, Corollary 5.4]. We conclude that the minimum of $q$ is attained at the point $z=a$, where it vanishes. This completes the proof.

This completes the proof in the interior case.

The boundary case ( $c f$. [4, Theorem 3.2])

We now consider the case that $\inf _{M} k\left(x_{0}, \cdot, t_{0}\right)$ occurs on the boundary of $\widehat{M}$; that is, $\underline{k}\left(x_{0}, t_{0}\right)=\mathcal{W}_{\left(x_{0}, t_{0}\right)}\left(y_{0}, y_{0}\right)$ for some $y_{0} \in S_{\left(x_{0}, t_{0}\right)} M$. Consider the function $K$ defined on $T M \times[0, T)$ by $K(x, y, t)=\mathcal{W}_{(x, t)}(y, y)$. Then the function $\Phi(x, y, t):=\phi(x, t) g_{(x, t)}(y, y)$ is a lower support for $K$ at $\left(x_{0}, y_{0}, t_{0}\right)$. In particular,

$$
\begin{aligned}
\partial_{t}(K-\Phi) & \leq 0, \\
\text { and } \widehat{\mathscr{L}}(K-\Phi) & \geq 0
\end{aligned}
$$

at $\left(x_{0}, y_{0}, t_{0}\right)$ for any elliptic operator $\widehat{\mathscr{L}}$ on $T M$. We require the operator project to $\mathscr{L}$ on the first factor (at least at the point $\left.\left(x_{0}, y_{0}, t_{0}\right)\right)$, which leads us to consider an operator $\widehat{\mathscr{L}}$ locally of the form $\widehat{\mathscr{L}}=\dot{F}_{x}^{i j}\left(\partial_{x}^{i}-\Lambda_{i}{ }^{p} \partial_{y} p\right)\left(\partial_{x}^{j}-\Lambda_{j}{ }^{q} \partial_{y} q\right)$, where $\left\{x^{i}, y^{i}\right\}_{i=1}^{n}$ are coordinates for $T M$ near $\left(x_{0}, y_{0}\right)$. We choose these coordinates such that $\left\{x^{i}\right\}_{i=1}^{n}$ are normal coordinates on $M$ (with respect to $g_{t_{0}}$ ) based at $x_{0}$ and $\left\{y^{i}\right\}_{i=1}^{n}$ are the corresponding fibre coordinates (defined by $(x, y)=\left(x, y^{i} \partial_{x^{i}}\right)$ for tangent vectors $(x, y)$ near $\left.\left(x_{0}, y_{0}\right)\right)$. Moreover, we may assume that $\left\{\left.\partial_{x^{i}}\right|_{x_{0}}\right\}_{i=1}^{n}$ is a basis of eigenvectors of $\mathcal{W}_{\left(x_{0}, t_{0}\right)}$ with $y_{0}=\left.\partial_{x^{1}}\right|_{x_{0}}$.

Writing locally $K-\Phi=y^{k} y^{l}\left(\mathcal{W}_{k l}-g_{k l}\right)$, we find

$$
\left(\partial_{x^{i}}-\Lambda_{i}{ }^{p} \partial_{y} p\right)(K-\Phi)=y^{k} y^{l}\left(\partial_{x^{i}} \mathcal{W}_{k l}-\partial_{x^{i}} \phi g_{k l}\right)-2 \Lambda_{i}{ }^{p} y^{k}\left(\mathcal{W}_{k p}-\phi g_{k p}\right) \text {. }
$$

Thus, at the point $\left(x_{0}, y_{0}, t_{0}\right)$, we obtain

$$
0=\left(\partial_{x^{i}}-\Lambda_{i}^{p} \partial_{y} p\right)(K-\Phi)=\nabla_{i} \mathcal{W}_{11}-\nabla_{i} \phi
$$


We next compute

$$
\begin{aligned}
& \left(\partial_{x^{i}}-\Lambda_{i}{ }^{p} \partial_{y^{p}}\right)\left(\partial_{x^{j}}-\Lambda_{j}^{q} \partial_{y^{q}}\right)(K-\Phi) \\
& =y^{k} y^{l}\left(\partial_{x^{i}} \partial_{x^{j}} \mathcal{W}_{k l}-\partial_{x^{i}} \partial_{x^{j}} \phi g_{k l}-\partial_{x^{i}} \phi \partial_{x^{j}} g_{k l}-\partial_{x^{j}} \phi \partial_{x^{i}} g_{k l}-\phi \partial_{x^{i}} \partial_{x^{j}} g_{k l}\right) \\
& \quad-2 \Lambda_{j}^{q} y^{k}\left(\partial_{x^{i}} \mathcal{W}_{k q}-\partial_{x^{i}} \phi g_{k q}-\phi \partial_{x^{i}} g_{k p}\right) \\
& \quad-2 \Lambda_{i}{ }^{p} y^{k}\left(\partial_{x^{j}} \mathcal{W}_{k p}-\partial_{x^{j}} \phi g_{k p}-\phi \partial_{x^{j}} g_{k p}\right)+2 \Lambda_{i}{ }^{p} \Lambda_{j}{ }^{q}\left(\mathcal{W}_{p q}-\phi g_{p q}\right) .
\end{aligned}
$$

At the point $\left(x_{0}, y_{0}, t_{0}\right)$, we obtain

$$
\begin{aligned}
\widehat{\mathscr{L}}(K-\Phi)=\mathscr{L} \mathcal{W}_{11}-\mathscr{L} \phi & \\
-2 \dot{F}^{i j} & {\left[2 \Lambda_{i}{ }^{p}\left(\nabla_{j} \mathcal{W}_{1 p}-\nabla_{j} \mathcal{W}_{11} \delta_{1 p}\right)\right.} \\
& \left.-\Lambda_{i}{ }^{p} \Lambda_{j}{ }^{q}\left(\mathcal{W}_{p q}-\mathcal{W}_{11} \delta_{p q}\right)\right]
\end{aligned}
$$

Finally, we compute the time derivative

$$
\partial_{t}(K-\Phi)=y^{k} y^{l}\left(\partial_{t} \mathcal{W}_{k l}-\partial_{t} \phi g_{k l}-\phi \partial_{t} g_{k l}\right),
$$

which at $\left(x_{0}, y_{0}, t_{0}\right)$ becomes

$$
\partial_{t}(K-\Phi)=\partial_{t} \mathcal{W}_{11}-\mathcal{W}_{11} \partial_{t} g_{11}-\partial_{t} \phi .
$$

Let us recall the evolution equations for $\mathcal{W}$ and $g$ [2,3]:

$$
\begin{aligned}
\partial_{t} \mathcal{W}_{i j}=\mathscr{L} & \mathcal{W}_{i j}+\ddot{F}^{p q, r s} \nabla_{i} \mathcal{W}_{p q} \nabla_{j} \mathcal{W}_{r s}-2 \mathcal{W}_{i j}^{2} F \\
& +\left(|\mathcal{W}|_{F}^{2}-\sigma \operatorname{tr}(\dot{F})\right) \mathcal{W}_{i j}+2 \sigma F g_{i j}
\end{aligned}
$$

and

$$
\partial_{t} g_{i j}=-2 F \mathcal{W}_{i j}
$$

Putting (2.9) and (2.10) together, and applying the evolution equations (2.11) and (2.12), and the inequalities (2.8), we obtain

$$
\begin{aligned}
0 \geq & \left(\partial_{t}-\mathscr{L}\right)(K-\Phi)=-\left(\partial_{t}-\mathscr{L}\right) \phi+\left(|\mathcal{W}|_{F}^{2}-\sigma \operatorname{tr}(\dot{F})\right) \phi+2 \sigma F \\
& +\ddot{F}^{p q, r s} \nabla_{1} \mathcal{W}_{p q} \nabla_{1} \mathcal{W}_{r s} \\
& +2 \dot{F}^{i j} \Lambda_{i}{ }^{p}\left[2\left(\nabla_{j} \mathcal{W}_{1 p}-\nabla_{j} \mathcal{W}_{11} \delta_{1 p}\right)-\Lambda_{j}{ }^{q}\left(\mathcal{W}_{p q}-\mathcal{W}_{11} \delta_{p q}\right)\right]
\end{aligned}
$$

at the point $\left(x_{0}, y_{0}, t_{0}\right)$. Note that the term in the last line with $p=1$ vanishes.

Using a trick of Brendle [13, Proposition 8] (see also [8, Theorem 7]) we also obtain $\nabla_{1} \mathcal{W}_{11}=0$ at the point $\left(x_{0}, t_{0}\right)$ : 
Lemma 2.4. $\nabla_{1} \mathcal{W}_{11}$ vanishes at $\left(x_{0}, y_{0}, t_{0}\right)$.

Sketch proof of Lemma 2.4. Since $\kappa_{1}\left(x_{0}, t_{0}\right)=\inf _{y \neq x_{0}} k\left(x_{0}, y, t_{0}\right)$, we have $0 \leq Z\left(x_{0}, y, t_{0}\right):=2\left\langle X\left(x_{0}, t_{0}\right)-X\left(y, t_{0}\right), v\left(x_{0}, t_{0}\right)\right\rangle-\kappa_{1}\left(x_{0}, t_{0}\right)\left\|X\left(x_{0}, t_{0}\right)-X\left(y, t_{0}\right)\right\|^{2}$ for all $y \in M$. In particular, $0 \leq f(s):=Z\left(x_{0}, \gamma(s), t_{0}\right)$ for all $s$, where $\gamma(s):=$ $\exp _{x_{0}} s y_{0}$. It is straightforward to compute $0=f(0)=f^{\prime}(0)=f^{\prime \prime}(0)$, which, since $f \geq 0$, implies that $f^{\prime \prime \prime}(0)=0$. But a further straightforward computation yields $f^{\prime \prime \prime}(0)=2 \nabla_{1} \mathcal{W}_{11}$.

Applying the following proposition to (2.13) completes the proof.

Proposition 2.5. Let $f: \Gamma_{+} \rightarrow \mathbb{R}$ be a smooth, symmetric function which is monotone increasing in each variable and inverse-concave, and let $F: \mathcal{C}_{+} \rightarrow \mathbb{R}$ be the function defined on the cone $\mathcal{C}_{+}$of positive definite symmetric matrices by $F(A)=f\left(\lambda(A)\right.$ ), where $\lambda$ denotes the eigenvalue map. If $A \in \mathcal{C}_{+}$and $y$ is an eigenvector of A corresponding to its smallest eigenvalue, then, for any totally symmetric 3-tensor $T$ with $T(y, y, y)=0$, we have

$$
\begin{aligned}
0 \leq y^{i} y^{j} \ddot{F}^{p q, r s} T_{i p q} T_{j r s}+2 \dot{F}^{k l} \sup _{\Lambda}[ & 2 \Lambda_{k}^{p} y^{i}\left(T_{i l p}-y^{r} y^{s} T_{l r s} \delta_{i q}\right) \\
& \left.-\Lambda_{k}^{p} \Lambda_{l}^{q}\left(A_{p q}-y^{r} y^{s} A_{r s} \delta_{p q}\right)\right]
\end{aligned}
$$

at the matrix A. Moreover, equality holds only if $T(v, y, y)=0$ for all $v \in \mathbb{R}^{n}$.

Proof of Proposition 2.5. We first observe that it suffices to prove the claim for those $A \in \mathcal{C}_{+}$having distinct eigenvalues: The expression

$$
Q:=2 \dot{F}^{k l}\left[2 \Lambda_{k}^{p} y^{i}\left(T_{i l p}-y^{r} y^{s} T_{l r s} \delta_{i q}\right)-\Lambda_{k}^{p} \Lambda_{l}^{q}\left(A_{p q}-y^{r} y^{s} A_{r s} \delta_{p q}\right)\right]
$$

is continuous in $A$, and hence the supremum over $\Lambda$ is upper semi-continuous in $A$; so the general case follows by taking a sequence of matrices $A^{(k)} \in \mathcal{C}_{+}$approaching $A$ with each $A^{(k)}$ having distinct eigenvalues.

So suppose that $A$ has distinct eigenvalues and let $\left\{e_{i}\right\}_{i=1}^{n}$ be an orthonormal frame of eigenvectors of $A$ with $e_{1}=y$. Then

$$
Q=2 \dot{F}^{k l}\left[2 \Lambda_{k}^{p}\left(T_{1 l p}-T_{l 11} \delta_{1 q}\right)-\Lambda_{k}^{p} \Lambda_{l}^{q}\left(A_{p q}-A_{11} \delta_{p q}\right)\right] .
$$

Observe that the supremum over $\Lambda$ occurs when $\Lambda_{l q}=\left(\lambda_{q}-\lambda_{1}\right)^{-1} T_{1 l q}$ for $i, p>1$. With this choice, we obtain

$$
Q=2 \dot{F}^{k l} R^{p q} T_{1 k q} T_{1 l p},
$$


where $R^{p q}:=\left(\lambda_{p}-\lambda_{1}\right)^{-1} \delta^{p q}$ for $p, q \neq 1$ and zero otherwise. Therefore, it suffices to prove that

$$
0 \leq\left(\ddot{F}^{p q, r s}+2 \dot{F}^{p r} R^{q s}\right) B_{p q} B_{r s}
$$

for any symmetric $B$ with $B_{11}=0$ with equality only if $B_{1 q}=0$ for all $q$. The expression we want to estimate may be written in terms of the function $f$ as follows (see, for example, [4, Theorem 5.1]):

$$
\begin{aligned}
\left(\ddot{F}^{p q, r s}+2 \dot{F}^{p r} R^{q s}\right) B_{p q} B_{r s}= & \ddot{f}^{p q} B_{p p} B_{q q}+\sum_{p \neq q} \frac{\dot{f}^{p}-\dot{f}^{q}}{\lambda_{p}-\lambda_{q}} B_{p q}^{2}+2 \sum_{p=1, q=2}^{n} \frac{\dot{f}^{p}}{\lambda_{q}-\lambda_{1}} B_{p q}^{2} \\
= & \ddot{f}^{p q} B_{p p} B_{q q}+2 \sum_{p>1, q>1} \frac{\dot{f}^{p} \delta^{p q}}{\lambda_{p}-\lambda_{1}} B_{p p} B_{q q} \\
& +\sum_{p \neq q} \frac{\dot{f}^{p}-\dot{f}^{q}}{\lambda_{p}-\lambda_{q}} B_{p q}^{2} \\
& +2 \sum_{p=2}^{n} \frac{\dot{f}^{1}}{\lambda_{p}-\lambda_{1}} B_{p 1}^{2}+2 \sum_{\substack{p>1, q>1, p \neq q}} \frac{\dot{f}^{p}}{\lambda_{q}-\lambda_{1}} B_{p q}^{2} .
\end{aligned}
$$

We first estimate

$$
\begin{aligned}
\ddot{f}^{p q} B_{p p} B_{q q}+2 \sum_{p>1, q>1} \frac{\dot{f}^{p} \delta^{p q}}{\lambda_{p}-\lambda_{1}} B_{p p} B_{q q} & \geq \ddot{f}^{p q} B_{p p} B_{q q}+2 \sum_{p=2, q=2}^{n} \frac{\dot{f}^{p}}{\lambda_{p}} \delta^{p q} B_{p p} B_{q q} \\
& =\left(\ddot{f}^{p q}+2 \frac{\dot{f}^{p}}{\lambda_{p}} \delta^{p q}\right) B_{p p} B_{q q} \geq 0,
\end{aligned}
$$

where the final inequality follows from inverse-concavity of $f[4$, Theorem 2.1$]$. The remaining terms are

$$
\begin{aligned}
& \sum_{p \neq q} \frac{\dot{f}^{p}-\dot{f}^{q}}{\lambda_{p}-\lambda_{q}} B_{p q}^{2}+2 \sum_{p=2}^{n} \frac{\dot{f}^{1}}{\lambda_{p}-\lambda_{1}} B_{p 1}^{2}+2 \sum_{\substack{p>1, q>1, p \neq q}} \frac{\dot{f}^{p}}{\lambda_{q}-\lambda_{1}} B_{p q}^{2} \\
& =\sum_{\substack{p>1, q>1, p \neq q}}\left(\frac{\dot{f}^{p}-\dot{f}^{q}}{\lambda_{p}-\lambda_{q}}+2 \frac{\dot{f}^{p}}{\lambda_{q}-\lambda_{1}}\right) B_{p q}^{2}+2 \sum_{p=2}^{n}\left(\frac{\dot{f}^{p}-\dot{f}^{1}}{\lambda_{p}-\lambda_{1}}+\frac{\dot{f}^{1}}{\lambda_{p}-\lambda_{1}}\right) B_{p 1}^{2} \\
& \geq \sum_{\substack{p>1, q>1, p \neq q}}\left(\frac{\dot{f}^{p}-\dot{f}^{q}}{\lambda_{p}-\lambda_{q}}+\frac{\dot{f}^{p}}{\lambda_{q}}+\frac{\dot{f}^{q}}{\lambda_{p}}\right) B_{p q}^{2}+2 \sum_{p=2}^{n}\left(\frac{\dot{f}^{p}}{\lambda_{p}-\lambda_{1}}\right) B_{p 1}^{2} .
\end{aligned}
$$

The first term is non-negative by inverse-concavity of $f$ [4, Corollary 5.4] and the second term is clearly non-negative and vanishes only if $B_{1 q}=0$ for all $q>1$. This completes the proof.

This completes the proof that $\underline{k}$ is a viscosity supersolution of (2.1). 
Since the speed function satisfies (2.1), the statement of Theorem 1.2 follows from a simple comparison argument for viscosity solutions $(c f .[6,11])$ :

Define $\varphi(t):=\mathrm{e}^{2 \sigma \eta t}\left(\inf _{M \times\{t\}} \underline{k} / F-1 / \eta\right)$, where $\eta>0$ is such that $\sigma \eta \geq$ $\sigma \operatorname{tr}(\dot{F})$; that is, $\eta \geq \operatorname{tr}(\dot{F})$ for flows in the sphere, $\eta \leq \operatorname{tr}(\dot{F})$ for flows in hyperbolic space, and $\eta>0$ for flows in Euclidean space. We claim that $\varphi$ is non-decreasing. It suffices to prove that $\underline{k}(\cdot, t)-\left(1 / \eta+\mathrm{e}^{-2 \sigma \eta t} \varphi\left(t_{0}\right)-\varepsilon \mathrm{e}^{L\left(t-t_{0}\right)}\right) F(\cdot, t)>0$ for any $t_{0} \in[0, T), t \in\left[t_{0}, T\right)$ and any $\varepsilon>0$, where we have set $L:=1-2 \sigma \eta$. Taking $\varepsilon \rightarrow 0$ then gives $\underline{k}(\cdot, t)-\left(1 / \eta+\mathrm{e}^{-2 \sigma \eta t} \varphi\left(t_{0}\right)\right) F(\cdot, t) \geq 0$; that is, $\varphi(t) \geq \varphi\left(t_{0}\right)$ for all $t \geq t_{0}$ for any $t_{0}$. Now, at time $t_{0}$ we have $k\left(x, t_{0}\right)-$ $\left(1 / \eta+\mathrm{e}^{-2 \sigma \eta t_{0}} \varphi\left(t_{0}\right)-\varepsilon\right) F\left(x, t_{0}\right) \geq \varepsilon F\left(x, t_{0}\right)>0$. So suppose, contrary to the claim, that there is a point $\left(x_{1}, t_{1}\right) \in M \times\left[t_{0}, T\right)$ and some $\varepsilon>0$ such that $\underline{k}\left(x_{1}, t_{1}\right)-\left(1 / \eta+\mathrm{e}^{-2 \sigma \eta t_{1}} \varphi\left(t_{0}\right)-\varepsilon \mathrm{e}^{t_{1}-t_{0}}\right) F\left(x_{1}, t_{1}\right)=0$. Assuming that $t_{1}$ is the first such time, this means precisely that the function $\phi(x, t):=\left(1 / \eta+\mathrm{e}^{-2 \sigma \eta t} \varphi\left(t_{0}\right)-\right.$ $\left.\varepsilon \mathrm{e}^{L\left(t-t_{0}\right)}\right) F(x, t)$ is a lower support for $\underline{k}$ at $\left(x_{1}, t_{1}\right)$. But $\underline{k}$ is a viscosity supersolution of (2.1), so that, at the point $\left(x_{1}, t_{1}\right), \phi$ satisfies

$$
\begin{aligned}
0 \geq & -\left(\partial_{t}-\mathscr{L}\right) \phi+\left(|\mathcal{W}|_{F}^{2}-\sigma \operatorname{tr}(\dot{F})\right) \phi+2 \sigma F \\
= & \left(2 \eta \sigma \mathrm{e}^{-2 \sigma \eta t_{1}} \varphi\left(t_{0}\right)+L \varepsilon \mathrm{e}^{L\left(t_{1}-t_{0}\right)}\right) F \\
& -\left(\frac{1}{\eta}+\mathrm{e}^{-2 \sigma \eta t_{1}} \varphi\left(t_{0}\right)-\varepsilon \mathrm{e}^{L\left(t_{1}-t_{0}\right)}\right)\left(|\mathcal{W}|_{F}^{2}+\sigma \operatorname{tr}(\dot{F})\right) \\
& +\left(\frac{1}{\eta}+\mathrm{e}^{-2 \sigma \eta t_{1}} \varphi\left(t_{0}\right)-\varepsilon \mathrm{e}^{L\left(t_{1}-t_{0}\right)}\right) F\left(|\mathcal{W}|_{F}^{2}-\sigma \operatorname{tr}(\dot{F})\right)+2 \sigma F \\
= & 2 \mathrm{e}^{-2 \sigma \eta t_{1}} \varphi\left(t_{0}\right) F(\sigma \eta-\sigma \operatorname{tr}(\dot{F}))+\varepsilon \mathrm{e}^{L\left(t_{1}-t_{0}\right)} F(L+2 \sigma \operatorname{tr}(\dot{F})) \\
& +\frac{2}{\eta} F(\sigma \eta-\sigma \operatorname{tr}(\dot{F})) \\
\geq & \varepsilon \mathrm{e}^{L\left(t_{1}-t_{0}\right)}>0
\end{aligned}
$$

where we used $L:=1-2 \sigma \eta$, and $\sigma \eta \geq \sigma \operatorname{tr}(\dot{F})$ in the last line. This contradiction proves that $\phi$ could not have reached zero on $\left[t_{0}, T\right)$, which, as explained above, proves that $\varphi$ is non-decreasing. Therefore,

$$
\left(\frac{\underline{k}(x, t)}{F(x, t)}-\frac{1}{\eta}\right) \mathrm{e}^{2 \sigma \eta t} \geq \inf _{M \times\{t\}}\left(\frac{\underline{k}}{F}-\frac{1}{\eta}\right) \mathrm{e}^{2 \sigma \eta t}=: \varphi(t) \geq \varphi(0)=\inf _{M \times\{0\}}\left(\frac{\underline{k}}{F}-\frac{1}{\eta}\right) .
$$

This proves Theorem 1.2.

\section{Convex solutions in Euclidean space}

We now give an application of non-collapsing to flows of convex hypersurfaces; namely, we give a new proof that convex hypersurfaces contract to round points 
under the flow (CF) in Euclidean space when the speed is both concave and inverseconcave. This result was originally proved by the first author via a curvature pinching estimate [4].

We begin with some background results on fully non-linear curvature flows (CF). Given smooth, compact initial data on which $F$ is defined, we obtain unique solutions for a short time [2]. Since we can enclose the initial hypersurface by a large sphere, which shrinks to a point in finite time, the avoidance principle (see, for example, [6, Theorem 5]) implies that the maximal time $T$ of existence of the solution must be finite. For inverse-concave speeds, the non-collapsing estimate yields a preserved cone of curvatures for the flow, since $\kappa_{\min } \geq \underline{k} \geq k_{0} F$. This implies that the flow is uniformly parabolic, since positive bounds for $\dot{F}$ on the intersection of the preserved cone with the unit sphere $\{|\mathcal{W}|=1\}$ extend to bounds on the entire cone. If $F$ is also a concave function, then global regularity of solutions may be obtained by appealing to the scalar parabolic theory of Krylov-Safanov [22] and Evans and Krylov [18,21] (cf. [7,9]). We conclude that the solution will remain smooth until $\max _{M \times\{t\}} F \rightarrow \infty$ as $t \rightarrow T$.

The key to our proof of the convergence theorem is showing that the normalised interior and exterior ball curvatures improve to unity at a singularity. This is achieved using a blow-up argument and applying the strong maximum principle.

Theorem 3.1. Suppose $F$ is concave and inverse-concave. Then along any convex solution $X: M^{n} \times[0, T) \rightarrow \mathbb{R}^{n+1}$ of $(\mathrm{CF})$ the following estimates hold:

1 For every $\varepsilon>0$ there exists $F_{\varepsilon}<\infty$ such that

$$
F>F_{\varepsilon} \Rightarrow \bar{k} \leq(1+\varepsilon) F
$$

2 For every $\delta>0$ there exists $F_{\delta}<\infty$ such that

$$
F>F_{\delta} \quad \Rightarrow \quad \underline{k} \geq(1-\delta) F
$$

Proof. We will blow the solution up at a point where $F$ is becoming large. Applying the strong maximum principle, and making use of the gradient term appearing in (2.1), we find that this limit must be a shrinking sphere, from which the claims follow. We note that the only auxillary result we require is the fact that the only closed, convex, embedded hypersurfaces of $\mathbb{R}^{n}$ with $F$ constant are spheres [17]. When $F$ is the mean curvature, this is a well-known theorem of Alexandrov [1].

Suppose the first estimate were false. Then there exists a sequence $\left(x_{i}, t_{i}\right) \in$ $M \times[0, T)$ such that $F\left(x_{i}, t_{i}\right) \rightarrow \infty$ but $\frac{\bar{k}}{F}\left(x_{i}, t_{i}\right) \rightarrow\left(1+\varepsilon_{0}\right)$, where $\varepsilon_{0}>0$. By Theorem 1.2, $\varepsilon_{0}<\infty$. Set $\lambda_{i}:=F\left(x_{i}, t_{i}\right)$ and consider the blow-up sequence

$$
X_{i}(x, t):=\lambda_{i}\left(X\left(x, \lambda_{i}^{-2} t+t_{i}\right)-X_{i}\left(x_{i}, t_{i}\right)\right)
$$

It is easily checked that $X_{i}: M^{n} \times\left[-\lambda_{i}^{2} t_{i}, 0\right] \rightarrow \mathbb{R}^{n+1}$ is a solution of the flow (CF) for each $i$. Moreover, for each $i$, we have $\max _{M \times\left[-\lambda_{i}^{2} t_{i}, 0\right]} F_{i}=F_{i}\left(x_{i}, 0\right)=1$ and 
$X_{i}\left(x_{i}, 0\right)=0$. It follows that the sequence $X_{i}$ converges locally uniformly along a subsequence to a smooth limit flow $X_{\infty}: M_{\infty} \times(-\infty, 0] \rightarrow \mathbb{R}^{n+1}(c f .[12,14,15])$.

Since the ratio $\underline{k} / F$ is invariant under rescaling, we have

$$
\frac{\underline{k}_{i}}{F_{i}}\left(x_{i}, 0\right)=\frac{\underline{k}}{F}\left(x_{i}, t_{i}\right) \geq k_{0}>0,
$$

which implies that the image of each $X_{i}$ is contained in a compact set. It follows that the convergence is global, so that $M_{\infty} \cong M$.

We now show that $\underline{k} / F$ must be constant on the limit flow $X_{\infty}$; for if not, by Proposition 2.1 and the strong maximum principle (see, for example, [16]), its spatial maximum must must decrease monotonically, by an amount $L$ say, on some sub-interval $\left[t_{1}, t_{2}\right]$ of $(-\infty, 0]$. But then (passing to the convergent subsequence) there must exist sequences of times $t_{1, i}, t_{2, i} \in\left[-\lambda_{i}^{2} t_{i}, 0\right]$ with $t_{1, i} \rightarrow t_{1}$ and $t_{2, i} \rightarrow t_{2}$ such that

$$
L-\varepsilon \leq \max _{M} \frac{\bar{k}}{F}\left(\cdot, \lambda_{i}^{-2} t_{1, i}+t_{i}\right)-\max _{M} \frac{\bar{k}}{F}\left(\cdot, \lambda_{i}^{-2} t_{2, i}+t_{i}\right)
$$

for any $\varepsilon>0$, so long as $i$ is chosen accordingly large. But since $\lambda_{i} \rightarrow \infty$, the right-hand side of (3.1) converges to zero. It follows that $\sup _{M \times\{t\}} \bar{k} / F$ is independent of $t$. Since $M$ is compact, the space-time supremum of $\bar{k} / F$ is attained at an interior space-time point, and we deduce that $\bar{k} / F$ is constant. Since there is a sequence of points $x_{i}$ for which $\frac{\bar{k}_{i}}{F_{i}}\left(x_{i}, 0\right) \rightarrow\left(1+\varepsilon_{0}\right)$, we must have $\bar{k} \equiv\left(1+\varepsilon_{0}\right) F$ on the limit. In particular, we have $0 \equiv\left(\partial_{t}-\mathscr{L}\right)\left(\underline{k}-\left(1+\varepsilon_{0}\right) F\right)$. But then, computing as in Proposition 2.1, we find $0 \equiv \nabla \bar{k} \equiv\left(1+\varepsilon_{0}\right) \nabla F$ due to Propositions 2.3 and 2.5. But the only closed, convex hypersurfaces of $\mathbb{R}^{n}$ with $F$ constant are spheres [17], which satisfy $\bar{k} \equiv F$. This contradicts $\varepsilon_{0}>0$.

The proof of the second estimate is similar.

Remark 3.2. We note that, for flows by convex speed functions, where exterior non-collapsing holds, the proof of the exerior ball estimate goes through. However, for flows by concave speed functions, where interior non-collapsing holds, the proof of the interior ball estimate does not go through without some additional condition (such as a pinching condition) to ensure that the blow-up limit is convex. In fact, due to the examples constructed by Andrews-McCoy-Zheng [10, Section 5], one cannot expect such a result to hold in general.

We now prove the convergence result:

Theorem 3.3. Let $X: M^{n} \times[0, T) \rightarrow \mathbb{R}^{n+1}$ be a maximal solution of the curvature flow $(\mathrm{CF})$ such that the speed is a concave, inverse-concave function of the Weingarten map. Then $X$ converges to a constant $p \in \mathbb{R}^{n+1}$ as $t \rightarrow T$, and the rescaled embeddings

$$
\tilde{X}(x, t):=\frac{X(x, t)-p}{\sqrt{2(T-t)}}
$$

converge in $C^{2}$ as $t \rightarrow T$ to a limit embedding with image equal to the unit sphere $S^{n}$. 
Proof of Theorem 3.3. We first apply Theorem 1.2 to show that the solution converges uniformly to a point $p \in \mathbb{R}^{n+1}$ in the Hausdorff metric: Observe that $\left|X\left(x_{1}, t\right)-X\left(x_{2}, t\right)\right| \leq 2 r_{+}(t)$ for every $x_{1}, x_{2} \in M$ and every $t \in[0, T)$, where $r_{+}(t)$ denotes the circumradius of $X(M, t)$ (this is the radius of the smallest ball in $\mathbb{R}^{n+1}$ that contains the hypersurface $\left.X(M, t)\right)$. Since $X$ remains in the compact region enclosed by some initial circumsphere, it suffices to show that $r_{+} \rightarrow 0$ as $t \rightarrow T$. But this follows directly from Theorem 1.2: Since $\underline{k}(x, t)$ is the curvature of the smallest ball which encloses the hypersurface $X(M, t)$, and touches it at $X(x, t)$, we have

$$
\frac{1}{r_{+}} \geq \max _{M \times\{t\}} \underline{k} \geq k_{0} \max _{M \times\{t\}} F .
$$

But $\max _{M \times\{t\}} F \rightarrow \infty$.

We now deduce Hausdorff convergence of the rescaled hypersurfaces $\widetilde{X}(M, t)$ to the unit sphere: By Theorem (3.1), for all $\varepsilon>0$ there is a time $t_{\varepsilon} \in[0, T)$ such that $r_{+}(t) \leq(1+\varepsilon) r_{-}(t)$ for all $t \in\left[t_{\varepsilon}, T\right)$, where $r_{-}(t)$ is the in-radius of $X(M, t)$ (the radius of the largest ball enclosed by $X(M, t)$ ). By the avoidance principle the remaining time of existence at each time $t$ is no less than the lifespan of the shrinking sphere of initial radius $r_{-}(t)$, and no greater than the lifespan of the shrinking sphere of initial radius $r_{+}(t)$. These observations yield

$$
r_{-}(t) \leq \sqrt{2(T-t)} \leq r_{+}(t) \leq(1+\varepsilon) r_{-}(t)
$$

for all $t \in\left[t_{\varepsilon}, T\right)$. It follows that the circum- and in-radii of the rescaled solution approach unity as $t \rightarrow T$. We can also control the distance from the final point $p$ to the centre $p_{t}$ of any in-sphere of $X(M, t)$ : For any $t^{\prime} \in[t, T)$, the final point $p$ is enclosed by $X\left(M, t^{\prime}\right)$, which is enclosed by the sphere of radius $\sqrt{r_{+}(t)^{2}-2\left(t^{\prime}-t\right)}$ about $p_{t}$. Taking $t^{\prime} \rightarrow T$ and applying (3.2) gives

$$
\left|p-p_{t}\right| \leq \sqrt{r_{+}(t)^{2}-2(T-t)} \leq \sqrt{(1+\varepsilon)^{2} \cdot 2(T-t)-2(T-t)}
$$

for all $t \in\left[t_{\varepsilon}, T\right)$. Thus

$$
\frac{\left|p-p_{t}\right|}{\sqrt{2(T-t)}} \leq \sqrt{(1+\varepsilon)^{2}-1} .
$$

This yields the desired Hausdorff convergence of $\tilde{X}$ to the unit sphere.

Next we obtain bounds on the curvature of the rescaled flow $\widetilde{X}$ : Non-collapsing and the inequalities $r_{-}(t) \leq \sqrt{2(T-t)} \leq r_{+}(t)$ derived above yield

$$
\begin{aligned}
\frac{1}{\sqrt{2(T-t)}} & \leq \frac{1}{r_{-}(t)} \leq \min _{x \in M} \bar{k}(x, t) \\
& \leq k_{0} \min _{x \in M} F \leq \frac{k_{0}}{K_{0}} \min _{x \in M} \underline{k}(x, t) \leq \frac{k_{0}}{K_{0}} \min _{x \in M} \kappa_{\min }(x, t),
\end{aligned}
$$


and

$$
\begin{aligned}
\frac{1}{\sqrt{2(T-t)}} \geq \frac{1}{r_{+}(t)} & \geq \max _{x \in M} \underline{k}(x, t) \\
& \geq K_{0} \max _{x \in M} F \geq \frac{k_{0}}{K_{0}} \max _{x \in M} \bar{k}(x, t) \geq \frac{K_{0}}{k_{0}} \max _{x \in M} \kappa_{\max }(x, t) .
\end{aligned}
$$

By a well-known result of Hamilton [19, Lemma 14.2], this also implies convergence of the rescaled metrics, and we obtain the desired $C^{2}$-convergence.

Remark 3.4. One can obtain $C^{\infty}$-convergence in the above theorem by a standard bootstrapping procedure [20]. Namely, using the time-dependent curvature bounds, one obtains time-dependent bounds on the derivatives of the Weingarten map (of the underlying solution of the flow) to all orders from the curvature derivative estimates. Unfortunately, the resulting estimates do not quite have the right dependence on the remaining time. The correct dependence can be obtained using the interpolation inequality ( $c f$. [20, Section 9]). This yields exponential $C^{\infty}$-convergence of the corresponding solution of the normalised flow equation to the unit sphere ( $c f$. [20, Section 10]). By construction, this yields $C^{\infty}$-convergence of the rescaled solution.

\section{References}

[1] A. D. Alexandrov, A characteristic property of spheres, Ann. Mat. Pura Appl. (4) 58 (1962), 303-315.

[2] B. ANDREWS, Contraction of convex hypersurfaces in Euclidean space, Calc. Var. Partial Differential Equations 2 (1994), 151-171.

[3] B. ANDREWs, Contraction of convex hypersurfaces in Riemannian spaces, J. Differential Geom. 39 (1994), 407-431.

[4] B. ANDREWS, Pinching estimates and motion of hypersurfaces by curvature functions, J. Reine Angew. Math. 608 (2007), 17-33.

[5] B. ANDREWs, Noncollapsing in mean-convex mean curvature flow, Geom. Topol. 16 (2012), 1413-1418.

[6] B. ANDREWS, M. LANGFORD and J. MCCOY, Non-collapsing in fully non-linear curvature flows, Ann. Inst. H. Poincaré Anal. Non Linéaire 30 (2013), 23-32.

[7] B. ANDREWs, M. LANGFORD and J. MCCOY, Convexity estimates for fully non-linear surface flows, J. Differential Geom. 99 (2015), 47-75.

[8] B. ANDREWS and H. LI, Embedded constant mean curvature tori in the three-sphere, J. Differential Geom. 99 (2015), 169-189.

[9] B. ANDREWS and J. MCCOY, Convex hypersurfaces with pinched principal curvatures and flow of convex hypersurfaces by high powers of curvature, Trans. Amer. Math. Soc. 364 (2012), 3427-3447.

[10] B. AndREWs, J. MCCOY and Y. ZHENG, Contracting convex hypersurfaces by curvature, Calc. Var. Partial Differential Equations 47 (2013), 611-665.

[11] B. ANDrEws, X. HAN, H. LI and Y. WEI, Non-collapsing for hypersurface flows in the sphere and hyperbolic space, Ann. Sc. Norm. Super. Pisa Cl. Sci. (5) 14 (2015), 331-338.

[12] C. BAKER, "The Mean Curvature Flow of Submanifolds of High Codimension", PhD thesis, Australian National University 2010 
[13] S. BRENDLE, Embedded minimal tori in $S^{3}$ and the Lawson conjecture, Acta Math. 211 (2013), 177-190.

[14] P. BReuning, Compactness of immersions with local Lipschitz representation, Ann. Inst. Henri Poincaré, Anal. Non Linéaire 29 (2012), 545-572.

[15] A. A. COOPER, A compactness theorem for the second fundamental form, preprint, arXiv:1006.5697v4 [math.DG].

[16] F. DA LIO, Remarks on the strong maximum principle for viscosity solutions to fully nonlinear parabolic equations, Commun. Pure Appl. Anal. 3 (2004), 395-415.

[17] K. ECKER and G. HUISKEN, Immersed hypersurfaces with constant Weingarten curvature, Math. Ann. 283 (1989), 329-332.

[18] L. C. Evans, Classical solutions of fully nonlinear, convex, second-order elliptic equations, Comm. Pure Appl. Math. 35 (1982), 333-363.

[19] R. S. Hamilton, Three-manifolds with positive Ricci curvature, J. Differential Geom. 17 (1982), 255-306.

[20] G. HUISKEN, Flow by mean curvature of convex surfaces into spheres, J. Differential Geom. 20 (1984), 237-266.

[21] N. V. KRYLOv, Boundedly inhomogeneous elliptic and parabolic equations, Izv. Akad. Nauk SSSR Ser. Mat. 46 (1982), 487-523.

[22] N. V. KRYLOV and M. V. SAFONOV, A property of the solutions of parabolic equations with measurable coefficients, Izv. Akad. Nauk SSSR Ser. Mat. 44 (1980), 161-175, 239.

[23] W. SHENG and X.-J. WANG, Singularity profile in the mean curvature flow, Methods Appl. Anal. 16 (2009), 139-155.

Mathematical Sciences Institute Australian National University Acton ACT 2601, Australia ben.andrews@anu.edu.au mathew.langford@anu.edu.au 